\title{
Combined Cellular Automaton Model for Dynamic Recrystallization Evolution of 42 CrMo Cast Steel
}

Yi-Na Guo ${ }^{1,2^{*}} \mathbb{0}$, Yong-Tang Li2,3, Wen-Yan Tian ${ }^{1}$, Hui-Ping Qi ${ }^{2,3}$ and Hong-Hong Yan ${ }^{2,3}$

\begin{abstract}
The dynamic recrystallization (DRX) simulation performance largely depends on simulated grain topological structures. However, currently solutions used different models for describing two-dimensional (2D) and three-dimensional (3D) grain size distributions. Therefore, it is necessary to develop a more universal simulation technique. A cellular automaton (CA) model combined with an optimized topology deformation technology is proposed to simulate the microstructural evolution of $42 \mathrm{CrMo}$ cast steel during DRX. In order to obtain values of material constants adopted in the CA model, hot deformation characteristics of $42 \mathrm{CrMo}$ cast steel are investigated by hot compression metallographic testing. The proposed CA model deviates in two important aspects from the regular CA model. First, an optimized grain topology deformation technology is utilized for studying the hot compression effect on the topology of grain deformation. Second, the overlapping grain topological structures are optimized by using an independent component analysis method, and the influence of various thermomechanical parameters on the nucleation process, grain growth kinetics, and mean grain sizes observed during DRX are explored. Experimental study shows that the average relative root mean square error (RRMSE) of the mean grain diameter obtained by the regular CA model is equal to 0.173 , while the magnitude calculated using the proposed optimized CA model is only 0.11 . This paper proposes a novel combined CA model for simulating the microstructural evolution of $42 \mathrm{CrMo}$ cast steel, which notably uses a ICA-based grain topology deformation method to optimize the overlapping grain topological structures in simulation.
\end{abstract}

Keywords: 42CrMo cast steel, Dynamic recrystallization, Cellular automaton, Grain topology, Independent component analysis

\section{Introduction}

Grain growth and recrystallization are interactional processes depending on grain topology parameters and sizes. Due to the randomness and non-uniformity of grain orientations, simulated grain topological structures often exhibit overlapping problems, as shown in Figure 1 (the grain overlaps in the displayed three-dimensional (3D) and two-dimensional (2D) views are marked by the white circles).

\footnotetext{
*Correspondence: zulibest@163.com

${ }^{1}$ School of Electronic Information Engineering, Taiyuan University of Science and Technology, Taiyuan 030024, China

Full list of author information is available at the end of the article
}

However, a good grain topology model requires that grains fill the entire space and do not overlap with each other [1-3]. Many research groups around the world have conducted studies aiming at solving this problem. Using the 3D topology-related grain growth rate equation derived by Macpherson and Srolovitz [2], Wang et al. [3] from the University of Science and Technology Beijing suggested a 3D grain size model to describe quasi-stationary grain size distributions in 2008. In the same year, Pande and Cooper [4] from the United States Naval Research Laboratory presented a size-based continuum stochastic Fokker-Planck equation describing 2D grain size distributions, which confirmed the validity of the stochastic approach. In addition, Pande and Cooper [5] found in 2011 that Hillert's equation, 


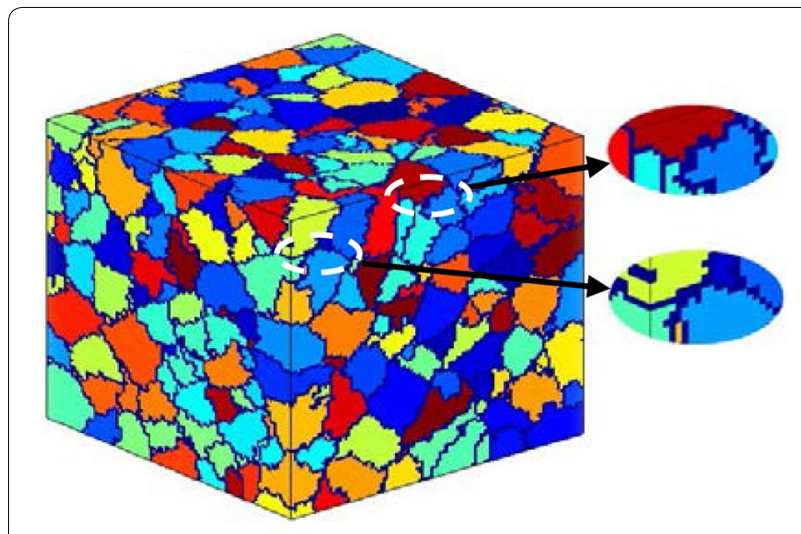

a

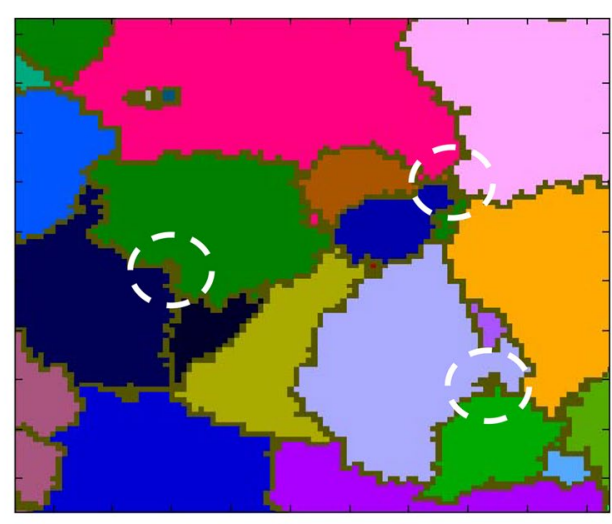

b

Figure 1 a 3D and $\mathbf{b} 2 \mathrm{D}$ simulated topological structures illustrating the grain overlap problem

Rayleigh's equation, and Fokker-Planck's continuity equation were not suitable for characterizing the upper time limits of the grain size distributions obtained during 2D grain growth in thin films. In 2012, Tucker et al. [6] from the Carnegie Mellon University tried to bridge the gap between the 2D and 3D grain size distributions by using Saltykov's method. It corrected the upper tail disparity between the 2D and 3D grain size distributions, but did not improve its lower tail. In 2015, Beers et al. [7] consolidated various approaches for characterizing grain boundaries in order to develop a multi-scale model of the initial GB structure and energy. In another study conducted by Keshavarz and Ghosh [8] during the same year, a hierarchical crystal model was proposed for simulating polycrystalline microstructures of Ni-based superalloys on the sub-grain, grain, and polycrystalline scales. The above-mentioned studies were indeed capable of solving the overlap problem of grain topological structures; however, they adopted different models for describing the $2 \mathrm{D}$ and $3 \mathrm{D}$ grain size distributions and thus could not be considered universal methods.
Independent component analysis (ICA) is a statistical and computational technique utilized for revealing hidden factors that underlie sets of random variables, measurements, or signals [9]. It has become one of the hottest topics in the areas of neural networks, advanced statistics, and signal processing in recent years. The distinctive advantage of ICA is that it is capable of finding components, which are both statistically independent and non-Gaussian (the statistical independence here refers to the components, which are mutually independent in the $2 \mathrm{D}$ and $3 \mathrm{D}$ spaces) $[10,11]$. As a result, the described method is expected to solve overlap problems of grain topological structures in a multidimensional space [12].

A cellular automation (CA) model combined with an optimized topology deformation technology is proposed to simulate the microstructural evolution of $42 \mathrm{CrMo}$ cast steel during dynamic recrystallization (DRX) and solve the problem of the overlapping reduction of grain topological structures during thermomechanical processing. A CA theoretical description of the related thermodynamic mechanism, CA state transition rules aimed at solving the grain overlap problem, an optimized ICAbased grain topology deformation technique, and a DRX CA process flowchart are provided in Section 2. Section 3 contains the analysis and discussion of the effects of temperature, strain rate, and cellular automaton steps (CAS) on the grain size evolution as well as the influence of the thermomechanical parameters on the nucleation process, grain growth kinetics, and mean grain sizes observed during DRX.

\section{Proposed Cellular Automaton Model}

\subsection{DRX Theoretical Modeling}

The CA method can be used to simulate the growth of newly recrystallized equiaxial grains, calculate the DRX nucleation rate, and describe the dislocation density variation and growth kinetics of dynamically recrystallized grains (all these processes are closely associated with actual hot deformation parameters). The nucleation and grain growth are two important aspects of DRX that affect material microstructure and are closely related to the dislocation density.

The following two assumptions developed by Ding et al. [13] are utilized for constructing the CA model:

(1) The initial dislocation density of all primary grains is uniform and equal to $10^{9} / \mathrm{m}^{2}$. When the dislocation density reaches a critical value, DRX occurs.

(2) The DRX nucleation occurs only at grain boundaries. Hence, only interface cells can become possible nucleation sites. 


\subsubsection{Dislocation Evolution}

The dislocation evolution during DRX mainly includes work hardening, dynamic recovery, and recrystallization processes. A phenomenological approach (the KM model) is utilized to predict variations of the dislocation density with strain $[14,15]$

$$
\frac{\mathrm{d} \rho}{\mathrm{d} \varepsilon}=k_{1} \rho^{\frac{1}{2}}-k_{2} \rho,
$$

where $\rho$ represents the cell dislocation evolution, $k_{1}=\frac{2 \theta_{0}}{\alpha u b}$ is the work hardening parameter, and $k_{2}=\frac{2 \theta_{0}}{\sigma_{s}}$ is the softening parameter. $\alpha$ is the dislocation interaction term, which is normally set to 0.5 for metals, $\mu$ is the shear modulus, and $b$ is the Burgers vector mode. $\theta_{0}$ is the hardening rate, which can be obtained from different values of strain rate $\dot{\varepsilon}$ and temperature $T$ ranging from $0.05 \mathrm{~s}^{-1}$ to $5 \mathrm{~s}^{-1}$ (for $\dot{\varepsilon}$ ) and from $850{ }^{\circ} \mathrm{C}$ to $1150{ }^{\circ} \mathrm{C}$ (for $T) . \sigma_{\mathrm{s}}$ is the saturated stress calculated by using the following equation

$$
\dot{\varepsilon}=A \sigma_{s}^{n} \exp \left(-\frac{Q_{a c t}}{R T}\right) .
$$

Using previous experimental and calculation results, the values of $A$ and $n$ are set to $1.484 \times 10^{15}$ and 5.619, respectively.

\subsubsection{Nucleation Rate}

In our proposed model, the nuclei formation during DRX is observed at primary and dynamically recrystallized grain boundaries, which is similar to the model developed by Ding et al. [13]. The DRX nucleation rate per unit grain boundary area can be expressed as a function of both temperature and strain rate by using the following equation

$$
\dot{n}(\dot{\varepsilon}, T)=C \dot{\varepsilon}^{m} \exp \left(-\frac{Q_{a c t}}{R T}\right),
$$

where $\dot{\varepsilon}$ is the strain rate, $\dot{n}$ is the nucleation rate, $C$ is the material constant, $Q_{a c t}$ is the nucleation activation energy, and the exponent $m$ is set to $1 . R$ is the molar gas constant, and $T$ is the temperature.

The critical dislocation density for the grain boundary nucleation can be calculated by taking into account the energy change, as proposed by Roberts and Ahlblom [15]:

$$
\rho_{c}=\left(\frac{20 \gamma \dot{\varepsilon}}{3 b l M \tau^{2}}\right)^{\frac{1}{3}},
$$

where $\tau=\frac{\mu b^{2}}{2}$ is the dislocation line energy, and $l=\frac{K_{1} \mu b}{\sigma}$ is the dislocation mean free path. $K_{1}$ is the constant equal to about 10 for metals, $\gamma$ is the grain boundary energy, and $M$ is the grain boundary mobility.

\subsubsection{Grain Growth Modeling}

The dislocation density of the newly recrystallized grains is much smaller than that of the initial grains due to DRX. The driving force for the DRX grain growth originates from the dislocation density difference between the dynamically recrystallized and initial grains. The relationship between the growth velocity $V$ and the driving force $f$ can be described by the following formula [14]:

$$
V=\lambda M f,
$$

where $\lambda$ is the hinder parameter for an alloy element. The driving force per unit area is $f=\tau\left(\rho_{m}-\rho_{i j}\right)-\frac{2 \gamma}{r}$. $\tau=\frac{\mu b^{2}}{2}$ is the dislocation line energy, $\rho_{m}$ is the dislocation density of parent grains, $\rho_{i j}$ is the cell dislocation evolution parameter with coordinates $i, j$, and $r$ is the grain radius. The grain boundary mobility $M$ can be calculated from the equation provided by Chen and Cui [1]:

$$
M=\frac{\delta D_{o b} b}{K T} \exp \left(-\frac{Q_{b}}{R T}\right),
$$

where $\delta$ is the characteristic grain boundary thickness, $D_{o b}$ is the boundary self-diffusion coefficient at absolute zero, $Q_{b}$ is the boundary diffusion activation energy, and $K$ is Boltzmann's constant.

The grain boundary energy $\gamma$ can be expressed as a function of misorientation by using the Read-Shockley equation [12]

$$
\gamma=\left\{\begin{array}{cc}
\gamma_{m} \frac{\theta}{\theta_{m}}\left(1-\operatorname{In} \frac{\theta}{\theta_{m}}\right), \theta<15^{\circ}, \\
\gamma_{m}, \quad \theta \geq 15^{\circ},
\end{array}\right.
$$

where $\theta$ is the grain boundary misorientation angle, and $\theta_{m}=15^{\circ}$ is the misorientation limit for low-angle boundaries. $\gamma_{m}=\frac{\mu b \theta_{m}}{4 \pi(1-v)}$ is the boundary energy, and $v$ is Poisson's ratio.

\subsection{CA Rules for Initial Microstructure Generation}

In accordance with the results of previous studies reported by Liu et al. [16], Hua et al. [17], Guan et al. [18], and Chen et al. [1, 14], which take into account the problem of grain boundary overlapping, the following CA state transition rules for austenitic grain growth before DRX were established based on the related 
thermodynamic movement mechanism, activation energy values, and curvature-driven mechanism.

Rule 1: Considering the temperature dependence of the austenitic grain growth, the cell orientation changes when the energy of grain boundary cells exceeds the activation barrier value. The probability of this event can be described by the following expression:

$$
P_{i}=C \frac{T-T_{A c i}}{T_{m}-T_{A c i}} \exp \left(-\frac{Q_{b}}{R T}\right),
$$

where $T$ is the current austenitizing temperature, $T_{A c i}$ is the initial austenitizing temperature, $T_{m}$ is the material melting point, and $Q_{b}$ is the boundary diffusion activation energy. The value of constant $C$ can be determined by making $T$ equal to $T_{m}$ and $P_{i}$ equal to 1 .

Rule 2: As shown clearly in Figure 2, if five or more neighbors of cell $C_{j}$ during the current CAS have the same state according to Moore's classification, the state of cell $C_{j}$ assumes that of its neighboring cells during the next CAS.

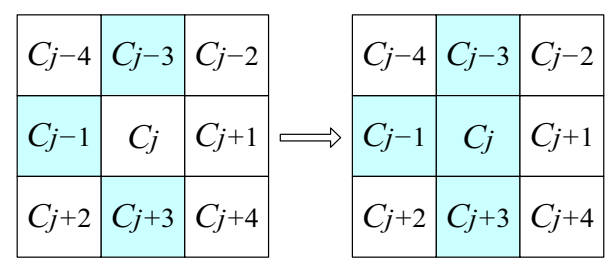

Figure 3 Schematic 2 of the $\xi_{C j}^{t} \rightarrow \xi_{C j}^{t+\Delta t}$ grain boundary movement

of the Hamilton function can be described by the following formula:

$$
\Delta E_{j \rightarrow k}=E_{k}-E_{j}=J \sum_{n}^{R}\left(1-\delta_{D k D n}\right)-J \sum_{n}^{R}\left(1-\delta_{D j D n}\right)
$$

where $J$ is the grain boundary measurement parameter, which is normally set to 1 , and $\delta$ is the Kronecker symbol. $R$ is the total number of neighbors for cell $C_{j}, n$ is the $n$th neighbor of cell $C_{j}, D_{j}$ is the orientation number for cell

$$
\xi_{C j}^{t+\Delta t}=f\left(\xi_{C j-4}^{t}, \xi_{C j-3}^{t}, \xi_{C j-2}^{t}, \xi_{C j-1}^{t}, \xi_{C j}^{t}, \xi_{C j+1}^{t}, \xi_{C j+2}^{t}, \xi_{C j+3}^{t}, \xi_{C j+4}^{t}\right)
$$

Rule 3: In Figure 3, if the states of any three $C_{j-3}, C_{j-1}$, $C_{j+1}$, or $C_{j+3}$ neighbors of cell $C_{j}$ have the same state during the current CAS, the state of cell $C_{j}$ assumes that of its neighboring cells during the next CAS.

$$
\xi_{C j}^{t+\Delta t}=f\left(\xi_{C j-3}^{t}, \xi_{C j-1}^{t}, \xi_{C j+1}^{t}, \xi_{C j+3}^{t}\right)
$$

Rule 4: In Figure 4, if any three $C_{j-4}, C_{j-2}, C_{j+2}$, or $C_{j+4}$ neighbors of cell $C_{j}$ have the same state during the current CAS, the state of cell $C_{j}$ assumes that of its neighboring cells during the next CAS.

$$
\xi_{C j}^{t+\Delta t}=f\left(\xi_{C j-4}^{t}, \xi_{C j-2}^{t}, \xi_{C j+2}^{t}, \xi_{C j+4}^{t}\right)
$$

Rule 5: If neither of the previous rules apply, the state of cell $C_{j}$ assumes the state of an optional neighboring cell $C_{k}$. The difference between the obtained grain boundary energies calculated from the deformation transformation
$C_{j}$, and $D_{n}$ is the $n$th neighbor orientation number for cell $C_{j}$.

If $\Delta E_{j \rightarrow k}<0$, the probability of the cell orientation change is 1 . If $\Delta E_{j \rightarrow k} \geq 0$, the probability of the cell orientation change is 0 .

$$
\begin{aligned}
\xi_{C j}^{t+\Delta t}= & f\left(\xi_{C k}^{t}\right), \\
& (k=j-4, j-3, j-2, j-1, j+1, j+2, j+3, j+4),
\end{aligned}
$$

Rule 6: For the purpose of making non-overlapping grains occupy the entire space, ICA is performed by optimizing grain topological structures until they become mutually independent. The correlation coefficient is used to evaluate the degree of grain cross-correlation. For example, when the correlation coefficient $r_{i j}$ for grains $i$ and $j$ is equal to 1 , the two grains overlap completely. When $r_{i j}$ equals 0 , the two grains are independent. When $0<\left|r_{i j}\right|<1$, the two grains partially overlap. When

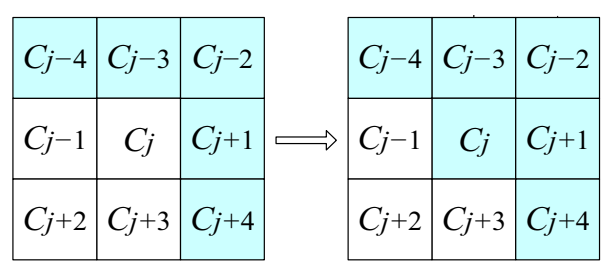

Figure 2 Schematic 1 of the $\xi_{C j}^{t} \rightarrow \xi_{C j}^{t+\Delta t}$ grain boundary movement

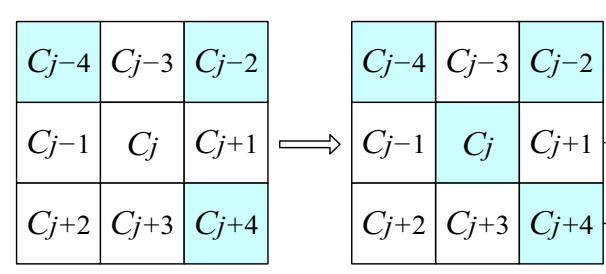

Figure 4 Schematic 3 of the $\xi_{C j}^{t} \rightarrow \xi_{C j}^{t+\Delta t}$ grain boundary movement 
$0<\left|r_{i j}\right|<0.3$, the two grains are weakly interdependent $[19,20]$.

If a particular cross-correlation criterion is not adapted, an error will occur during CA simulations. In order to minimize the influence of the cross-correlation factor on the simulation results and ensure computational efficiency and precision, we selected $0.1 \leq\left|r_{i j}\right| \leq 1,0.2 \leq\left|r_{i j}\right| \leq 1$, and $0.3 \leq\left|r_{i j}\right| \leq 1$ as grain cross-correlation criteria for testing the described CA model. If the correlation coefficient $r_{i j}$ meets the specified conditions, we assume that the grain overlap occurred and utilize the ICA method to mitigate it. Thus, the ICA-based grain topology deformation method and the related grain boundary mapping between two different coordinate systems are described in Section 2.3. The simulation error parameter $E_{r}=1-\frac{A_{\text {transf }}}{A_{0}}$ is used to characterize the mean correlation coefficient $\left|r_{\text {mean }}\right|$ (here $A_{0}$ and $A_{\text {transf }}$ are the grain areas before and after mapping, respectively). The grain area can be evaluated from the cell number for a particular grain [19].

As shown in Figure 5, the magnitudes of the error parameter $E_{r}$ calculated at different cross-correlation values increase with an increase in strain. The bigger is the lower limit of the cross-correlation criterion, the smaller is the error change with a strain increase. When $0.3 \leq\left|r_{i j}\right| \leq 1$, the obtained error values are close to zero. Therefore, we chose $0.3 \leq\left|r_{i j}\right| \leq 1$ as a cross-correlation criterion, which would be further utilized in this study.

Every 10 CAS (at $0.3 \leq\left|r_{i j}\right| \leq 1$ ), the ICA procedure is applied to ensure mutual independence between grains. The optimized grain topological structure can be described by

$$
C C^{*}=W_{m}^{\mathrm{T}} C C=\sum_{m} V^{\mathrm{T}}
$$

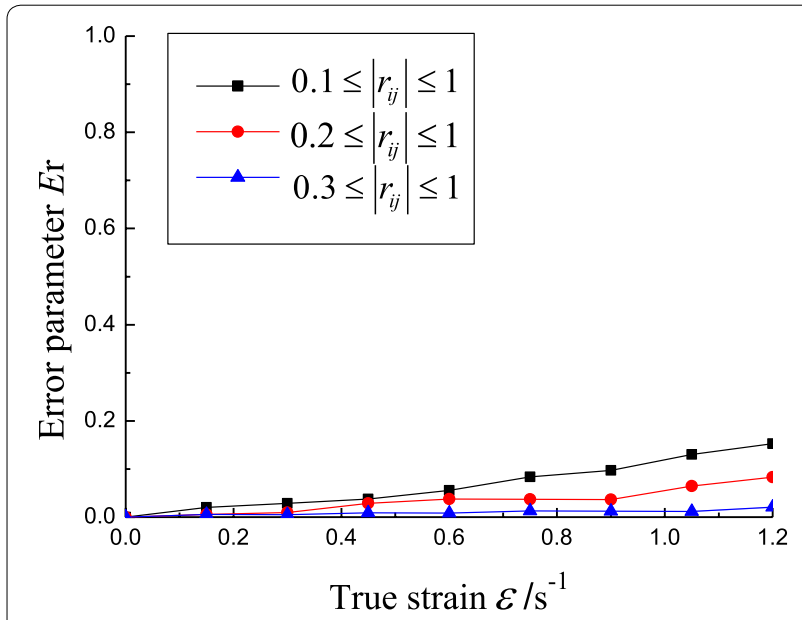

Figure 5 Eror parameter obtained at different strain and correlation coefficient values during grain boundary mapping where $C C$ is the $m \times n$ matrix of cell state variables, and the values of $m$ and $n$ are set to 400 each. $W_{m}$ is the singular value decomposition matrix of $m$ th order. $\sum_{m}=I_{m \times m} \sum$, where $I_{m \times m}$ is the square unit diagonal matrix of $m$ th order. $\Sigma$ is the $m \times n$ rectangular non-negative real diagonal matrix, and $V$ is the $C C^{\mathrm{T}} C C$ characteristic vector matrix of the $n$th order.

\subsection{ICA-based Grain Topology Deformation Method}

In 2012, Chen and Cui from Shanghai Jiao Tong University developed a new grain topology deformation technique with a double coordinate system, which was based on the previously used model $[1,14]$. The substance coordinate system was adopted to describe grain deformations and changes in cell sizes with an increase in strain. The cellular coordinate system was used to describe grain nucleation and growth of recrystallized grains (the related cell sizes were not affected by the strain increase). Such a model ensures that the new generation of recrystallized grains exhibit equiaxial growth and thus can describe the compression deformation process more accurately.

However, the majority of grain topological studies are focused on describing the 2D or 3D size distributions and do not offer a comprehensive solution for the problem of overlapping grains in a topological structure. In order to reduce the grain overlap and better arrange them to fill as much space as possible, an optimized topology deformation technique based on Chens' model and ICA is proposed in this study.

It is well known that, under certain conditions, new grains are formed at grain boundaries and grow in the equiaxial mode when the strain in the deformation area gradually approaches a critical value. Figure 6 describes the proposed topology deformation optimization model in more detail. In the substance coordinate system (shown in panel M1), the initial grains and grain boundaries are denoted by square cells. In panel M2, new grains and grain boundaries are formed with a strain increase. The dimensions of the deformed grains can be estimated according to $v_{x}=u e^{\varepsilon}$ and $v_{y}=u e^{-\varepsilon}$, where $u$ is the side length of a square cell, $\varepsilon$ is the strain, $v_{x}$ is the compressed long side, and $v_{y}$ is the compressed short side. During this process, thickening and overlapping of the grain boundaries inevitably occur. In panel M3, abnormal grain sizes and grain overlapping are mitigated by ICA; when the strain reaches the critical value, new grains are formed at the grain boundaries. In panel $\mathrm{C} 1$, the grain boundaries in the substance coordinate system are mapped to the cellular coordinate system to ensure that the recrystallized grains grow in the equiaxial mode. The abnormal grain shapes and grain boundaries observed during recrystallized grain growth in panel $\mathrm{C} 2$ are subsequently 


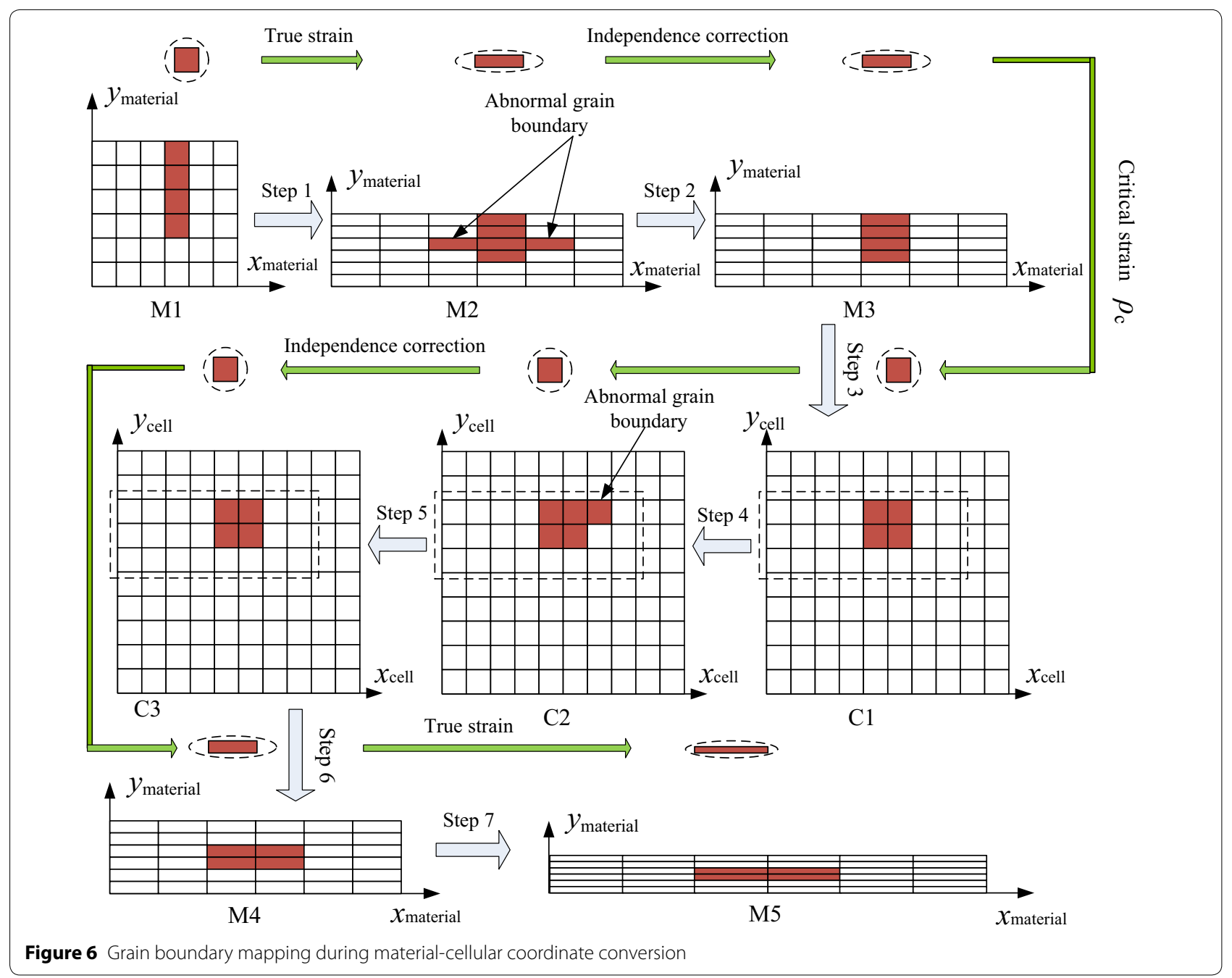

corrected by the ICA procedure depicted in panel C3. Panel M4 shows how the grain boundaries in the cellular coordinate system are mapped back to the substance coordinate system. In panel M5, new grains and grain boundaries are formed with a strain increase; as a result, the entire grain size changes followed by the next round of DRX.

\subsection{CA Model Flowchart for the DRX Process}

Figure 7 describes the CA model flowchart for the DRX process, which assumes normal grain growth in the initial topological structure. The dislocation density for the initial grain structure is set to $10^{9} / \mathrm{m}^{2}$. According to Kugler's suggestion made in 2004 [21], the shortest time of a cell growth is adopted as the CAS for the material and thermal deformation properties, which allows estimating the strain increment $\Delta \varepsilon_{C A}=\dot{\varepsilon} \cdot C A S$ and loop number $n=\frac{\varepsilon_{\text {total }}}{\Delta \varepsilon_{C A}}$ parameters for the DRX simulations $\left(\varepsilon_{\text {total }}\right.$ is the total strain). When the dislocation density $\rho_{i j}$ of a cell reaches the critical dislocation density $\rho_{c}$, the cell size in the substance coordinate system changes (provided that the sum of the strain increment $\sum \Delta \varepsilon_{C A}$ is proportional to the change in strain increment $\Delta \varepsilon_{\text {transf }}$ ). Afterwards, the grain boundaries in the substance coordinate system are mapped to the cellular coordinate system.

The cellular space is then scanned to update grain boundary variables and cell sizes. When cells are formed at the grain boundaries, the boundary variables are equal to 1 . When the cell dislocation density at the grain boundaries exceeds $\rho_{c}$, the cell recrystallization nucleation occurs. The initial dislocation density of the nucleated cells is set to $10^{-10} / \mathrm{m}^{2}$, and the initial grain orientations are selected randomly. The recrystallization number increases by 1 with every new nucleation event, 


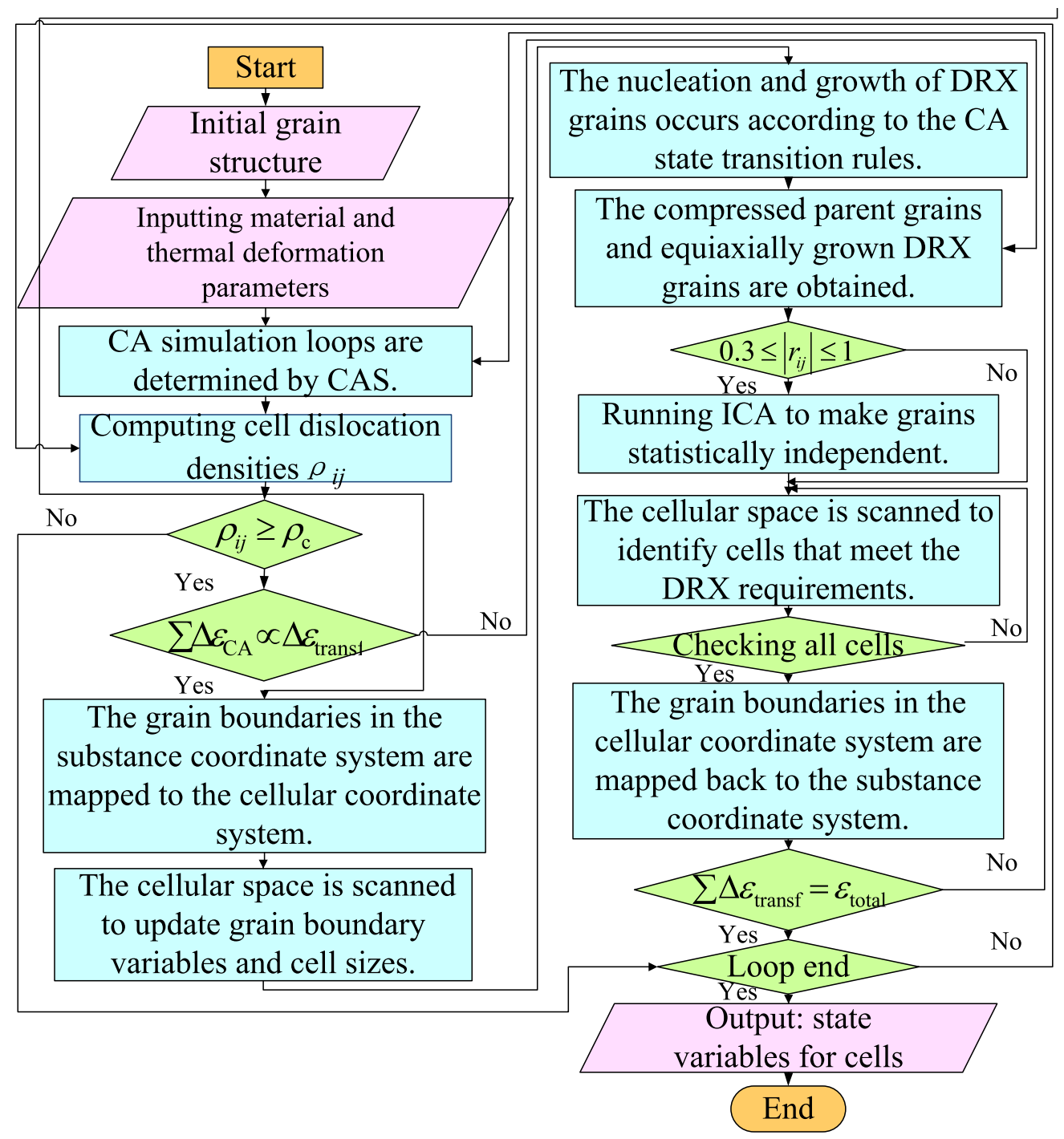

Figure 7 A flowchart describing the CA DRX model algorithm

while the DRX grains grow according to the CA state transition rules. As a result, compressed parent and equiaxially grown DRX grains are produced. When the grain correlation coefficients $\left|r_{i j}\right|$ of the cell state variables are in the range between 0.3 and 1, the ICA is activated to make the cells statistically independent. The $\left|r_{i j}\right|$ values can be determined by

$$
\left|r_{i j}\right|=\left|\frac{n \sum c_{i} c_{j}-\sum c_{i} \sum c_{j}}{\sqrt{n \sum c_{i}^{2}-\left(\sum c_{i}\right)^{2} \sqrt{n \sum c_{j}^{2}-\left(\sum c_{j}\right)^{2}}}}\right|,
$$

where $c_{i}$ and $c_{j}$ are the $i$ th and the $j$ th cell state variables, respectively, and $n$ is the grain internal cell number.
When the present cells do not meet the DRX requirements in the cellular space, the grain boundaries in the cellular coordinate system are mapped back to the substance coordinate system. If the change sum of the strain

Table 1 Material parameters of $42 \mathrm{CrMo}$ cast steel utilized for the DRX CA simulations

\begin{tabular}{lll}
\hline Material parameters & Units & Value \\
\hline Nucleation activation energy & $(\mathrm{kJ} / \mathrm{mol})$ & 389.247 \\
$\begin{array}{l}\text { Boundary diffusion activation energy } \\
\text { Boundary self-diffusion coefficient at } \\
\quad \begin{array}{l}\text { absolute zero } \\
\text { Shear modulus }\end{array}\end{array}$ & $\left(\mathrm{m}^{3} / \mathrm{s}\right)$ & $7.5 \times 10^{-13}$ \\
Burgers vector mode & $\mathrm{MPa}$ & $4.23 \times 10^{4}$ \\
\hline
\end{tabular}




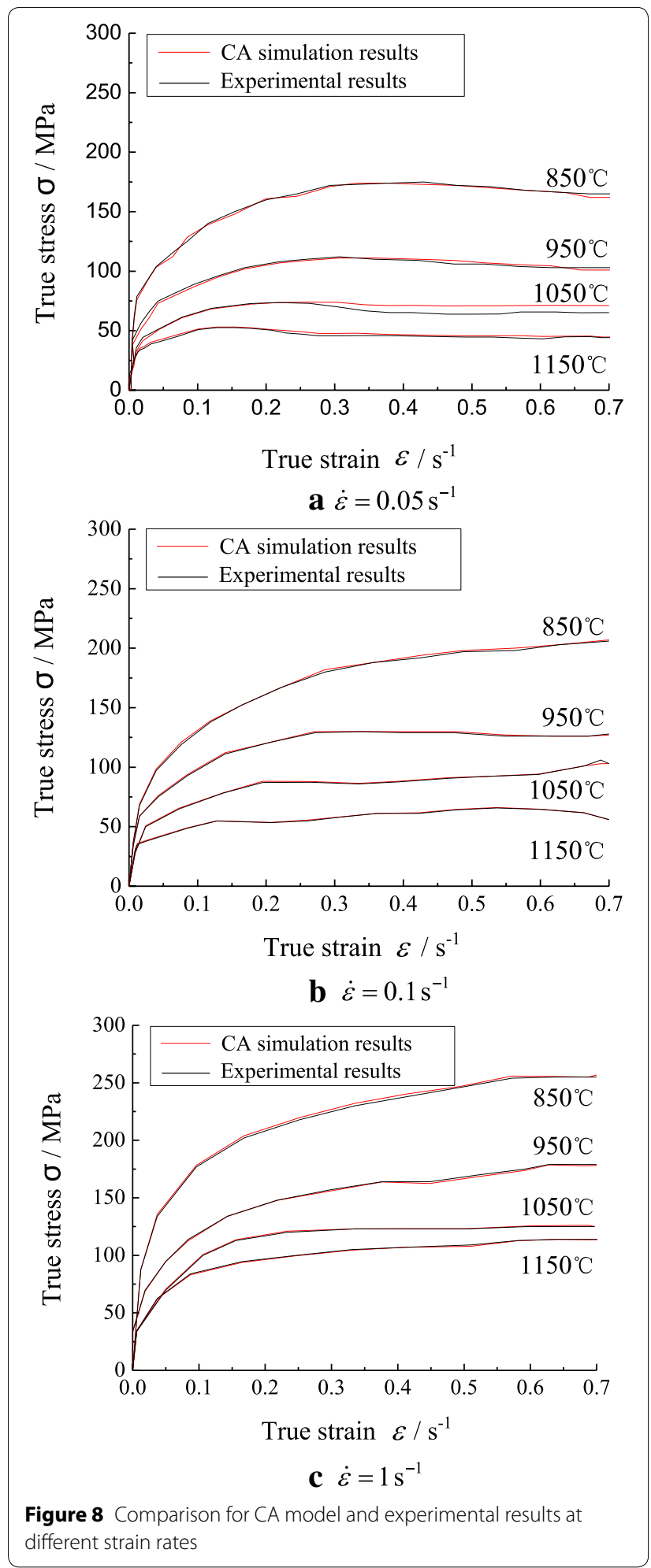

increment $\sum \Delta \varepsilon_{\text {transf }}$ is equal to the total strain value $\varepsilon_{\text {total }}$, the cell state variables are sent to the output indicating that the current simulation loop is complete.

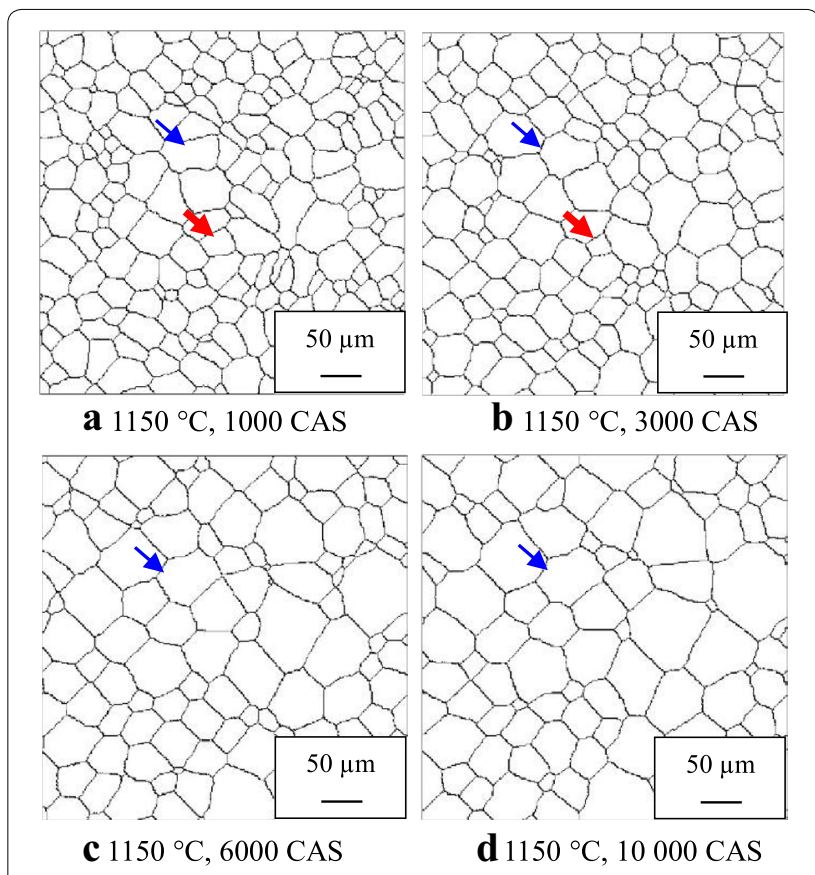

Figure 9 Grain microstructural evolution observed under different CA simulation conditions

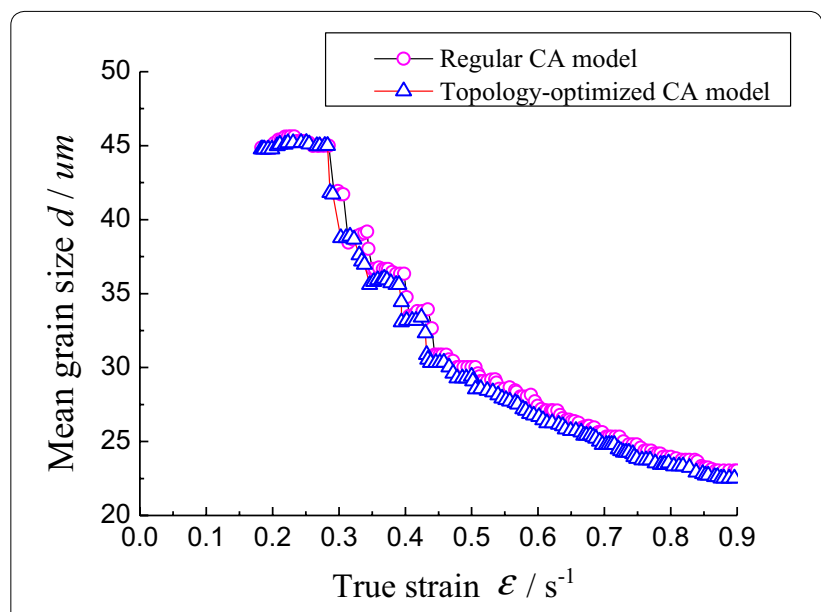

Figure 10 Comparison for the mean grain sizes of the CA models

\section{Results and Discussion}

In order to verify the accuracy and reliability of the proposed DRX CA model, the flow stress curves and microstructural evolution morphology of 42CrMo cast steel are selected as verification standards representing common DRX characteristics. The related material parameters of the DRX CA simulations are listed in Table $1[12,19]$.

As shown in Figure 8, the simulated values of the deformation temperature, strain rate, strain, and stress are in good agreement with the corresponding experimental 
(See figure on next page.)

Figure 11 Metallographic and CA simulated microstructures of $42 \mathrm{CrMo}$ steel obtained during thermomechanical processing at $\dot{\varepsilon}=0.05 \mathrm{~s}^{-1}$, $T=1050^{\circ} \mathrm{C}$, and different strain values

results obtained at all stages of the thermal deformation process.

The average root mean square errors (RMSEs) obtained at strain rates of $0.05 \mathrm{~s}^{-1}, 0.1 \mathrm{~s}^{-1}$, and $1 \mathrm{~s}^{-1}$ and temperatures of $850{ }^{\circ} \mathrm{C}, 950{ }^{\circ} \mathrm{C}, 1050{ }^{\circ} \mathrm{C}$, and $1150{ }^{\circ} \mathrm{C}$ are equal to $0.0612,0.0527$, and 0.0491 , respectively, indicating that the described CA model is capable of predicting experimental results with a relatively high accuracy.

The grain morphology produced during microstructural evolution is a major characteristic of the CA grain growth model. Figure 9 describes various CAS stages of the grain microstructural evolution obtained at a simulation temperature of $1150{ }^{\circ} \mathrm{C}$.

The larger is the CAS number, the larger is the average grain size and the smaller is the grain number. The size of the grain marked with the thin blue arrow increases gradually with an increase in the CAS number, whereas the size of the grain marked with the bold red arrow decreases with a CAS increment. In addition, smaller grains are always located around the grains with larger sizes and become eventually consumed by the latter during grain growth. The entire grain growth process is continuous and homogeneous and leads to an increase in the number of six-sided grains.

Figure 10 describes the differences in mean grain sizes obtained during regular and topology-optimized CA simulations. When the CA model with the optimized topology deformation technology is utilized, the DRX simulation speed is improved, while the recrystallized grain sizes decrease. At a strain of 0.9 , the mean grain diameter obtained by regular CA simulations is equal to $23.44 \mu \mathrm{m}$, while the mean grain diameter calculated using the proposed CA model is $23.22 \mu \mathrm{m}$ (the corresponding experimental value is equal to $23.08 \mu \mathrm{m})$. The average RRMSE of the mean grain diameter obtained by the comparable regular CA model simulations is 0.173 , whereas the RRMSE of the mean grain diameter obtained using the proposed CA model is only 0.11 . Thus, the simulation data produced by the proposed CA model better match the experimental results than the data obtained by the regular CA model.
Figure 11 describes the microstructures of 42CrMo cast steel obtained at a strain rate of $0.05 \mathrm{~s}^{-1}$, temperature of $1050{ }^{\circ} \mathrm{C}$, and strain values of $0.1,0.2,0.4,0.6$, and 0.9 .

The initial average grain diameter is set to $44.83 \mu \mathrm{m}$, which was previously obtained during metallographic studies of $42 \mathrm{CrMo}$ cast steel before DRX. When the strain value reaches 0.2 , recrystallization nucleation occurs, and new grains are formed at the grain boundaries. When the strain reaches 0.6 , the simulated mean grain diameter becomes $26.35 \mu \mathrm{m}$, while the corresponding experimental parameter is equal to $26.17 \mu \mathrm{m}$. The RRMSE of the mean grain diameter obtained via the proposed CA model simulation is 0.118 . When the strain value exceeds 0.6 , the simulated grain sizes become smaller and more homogeneous, which is in good agreement with the results of metallographic observations.

\section{Conclusions}

(1) Using the described thermodynamic grain growth and energy dissipation mechanisms, the ICA procedure and CA state transition rules consistent with the austenitic grain growth are presented, which can improve the simulation accuracy of initial grain topological structure before DRX.

(2) After taking into account the recrystallization nucleation, dislocation density, and grain growth parameters, the ICA method is utilized with the CA model, which can solve the grain overlap problem during CA simulations and optimize grain topological structures.

(3) Based on the flow stress-strain curves and the corresponding metallographs, the dynamically recrystallized CA state transition rules are established to investigate the hot compression effect on the grain deformation topology. It can obviously make the simulated grain topological structure during DRX is more approach to the experimental results.

(4) Compared with the regular CA model, the CA model combined with the optimized topology 

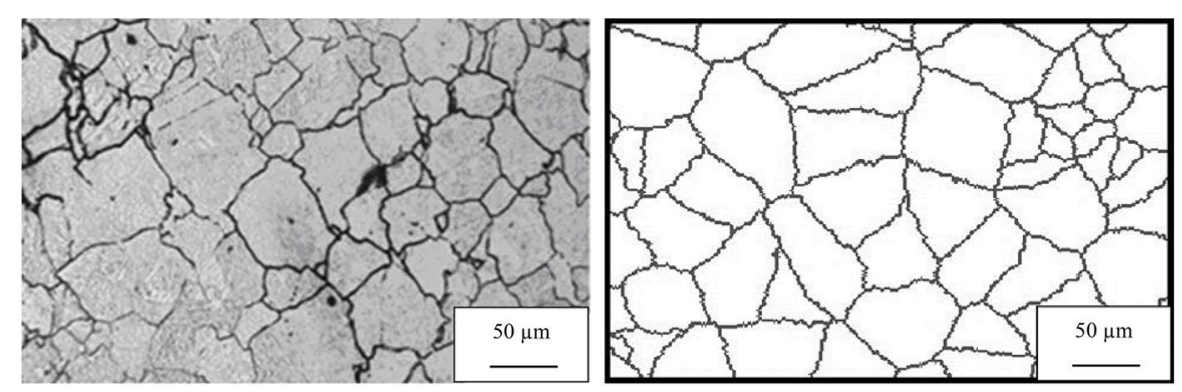

a $\varepsilon=0.1$
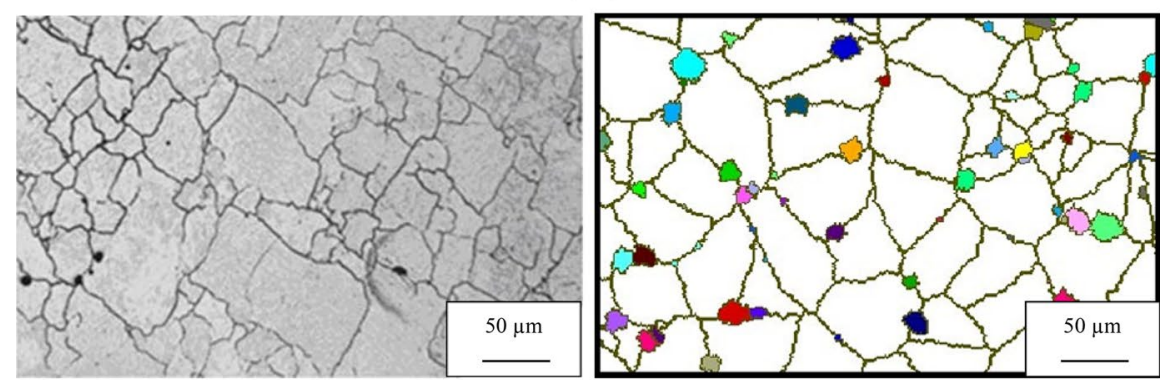

b $\varepsilon=0.2$

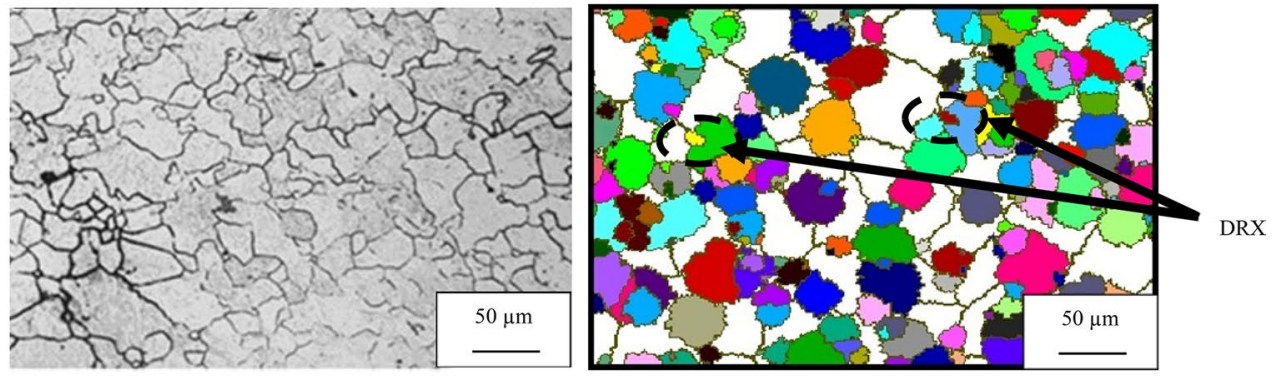

c $\varepsilon=0.4$
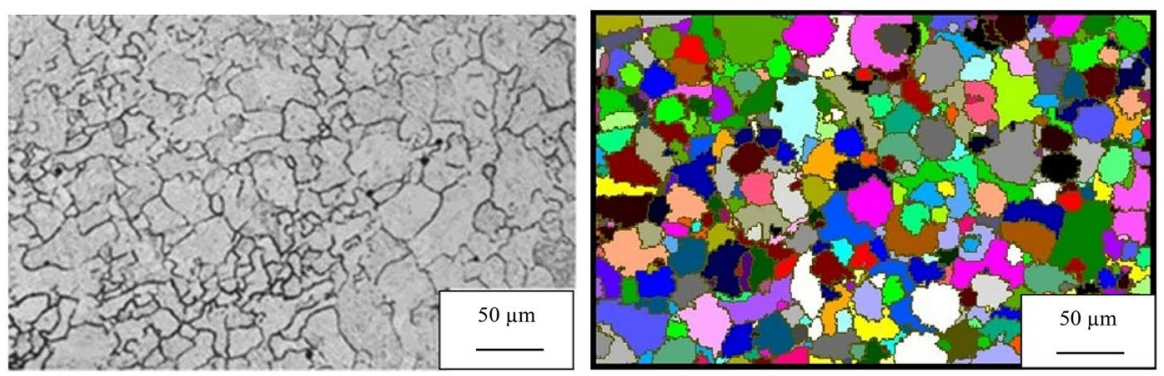

d $\varepsilon=0.6$
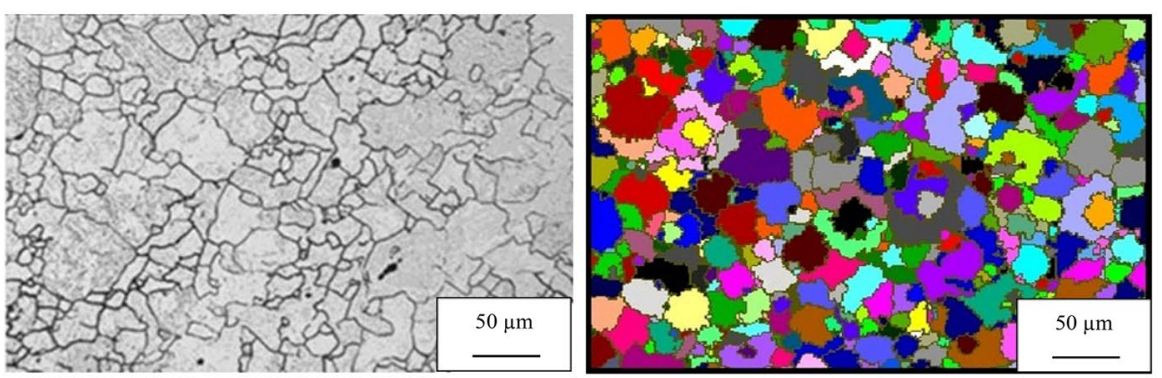

e $\varepsilon=0.9$ 
deformation technology can simulate the microstructural evolution of $42 \mathrm{CrMo}$ cast steel at different deformation parameters more accurately.

\begin{abstract}
Authors' Contributions
Y-NG was in charge of the whole trial; Y-NG and Y-TL wrote the manuscript; $\mathrm{H}-\mathrm{PQ}, \mathrm{W}-\mathrm{YT}$ and $\mathrm{H}-\mathrm{HY}$ assisted with sampling and laboratory analyses. All authors read and approved the final manuscript.
\end{abstract}

\section{Author Details}

${ }^{1}$ School of Electronic Information Engineering, Taiyuan University of Science and Technology, Taiyuan 030024, China. ${ }^{2}$ Shanxi Key Laboratory of Metallic Materials Forming Theory and Technology, Taiyuan 030024, China. ${ }^{3}$ School of Materials Science and Engineering, Taiyuan University of Science and Technology, Taiyuan 030024, China.

\section{Authors' Information}

Yi-Na Guo, born in 1981, is currently an associate professor at Taiyuan University of Science and Technology, China. Her research interests include digital simulation for precision forging forming technology and advanced manufacturing technology for precision forming.

Yong-Tang Li, born in 1957, is currently a professor and a PhD candidate supervisor at Taiyuan University of Science and Technology, China. He received his PhD degree from Tsinghua University, China, in 1994. His research interests include advanced technology for material manufacture and processing as well as hydraulic system modeling and simulations.

Wen-Yan Tian, born in 1983, is currently an associate professor at Taiyuan University of Science and Technology, China. She received her PhD degree from Sichuan University, China, in 2012. Her research interests include microwave measuring and energy applications.

Hui-Ping Qi, born in 1974, is an associate professor at Taiyuan University of Science and Technology, China. Her research interests include advanced manufacturing technology for material processing as well as successive plastic and precision forming

Hong-Hong Yan, born in 1979, is currently an associate professor at Taiyuan University of Science and Technology, China. Her research interests include advanced manufacturing technology for material processing, plastic successive and precision forming.

\section{Competing Interests}

The authors declare that they have no competing interests.

\section{Funding}

Supported by Key Program of National Natural Science Foundation of China (Grant No. 51135007), National Natural Science Foundation of China (Grant Nos. 51575371, 61301250), and Program for the Outstanding Innovative Teams of Higher Learning Institutions of Shanxi Province of China (Grant No. [2015]3).

\section{Publisher's Note}

Springer Nature remains neutral with regard to jurisdictional claims in published maps and institutional affiliations.

Received: 10 May 2016 Accepted: 21 September 2018 Published online: 08 October 2018

\section{References}

[1] F Chen, Z S Cui, J Liu, et al. Mesoscale simulation of the high-temperature austenitizing and dynamic recrystallization by coupling a Cellular Automaton with a topology deformation technique. Materials Science and Engineering A, 2010, 527(21): 5539-5549.

[2] R D MacPherson, D J Srolovitz. The von Neumann relation generalized to coarsening of three-dimensional microstructures. Nature, 2007, 446(7139): 1053-1055.

[3] H Wang, G Liu. Study of 3D quasi-stationary grain size distribution derived from macpherson-srolovitz topology-related grain growth rate equation. Acta Metallurgica Sinica, 2008, 44(7): 769-774. (in Chinese)
[4] C S Pande, K P Cooper. Self-similar grain size distribution in two dimensions: Analytical solution. Acta Materialia, 2008, 56(16): 4200-4205.

[5] C S Pande, K P Cooper. On the analytical solution for self-similar grain size distributions in two dimensions. Acta Materialia, 2011, 59(3): 955-961.

[6] J C Tucker, L H Chan, G S Rohere, et al. Comparison of grain size distributions in a Ni-based superalloy in three and two dimensions using the Saltykov method. Scripta Materialia, 2012, 66(8): 554-557.

[7] V P R M Beers, V G Kouznetsova, M G D Geers, et al. A multiscale model of grain boundary structure and energy: From atomistics to a continuum description. Acta Materialia, 2015, 82: 513-529.

[8] S Keshavarz, S Ghosh. Hierarchical crystal plasticity FE model for nickelbased superalloys: Sub-grain microstructures to polycrystalline aggregates. International Journal of Solids and Structures, 2015, 55: 17-31.

[9] H Aapo, K Juha, O Erkki. Independent component analysis. New York: John Wiley \& Sons, 2001

[10] Y Guo, S Huang, Y Li, et al. Edge effect elimination in single-mixture blind source separation. Circuits, Systems, and Signal Processing, 2013, (32)5: 2317-2334.

[11] Y Guo, S Huang, Y Li. Single-mixture source separation using dimensionality reduction of ensemble empirical mode decomposition and independent component analysis. Circuits, Systems, and Signal Processing, 2012, 31(6): 2047-2060.

[12] Y Guo, S Ding, Y Li, et al. Multiscale modeling for 42CrMo ring during blank-casting and rolling compound forming process. Journal of Mechanical Engineering, 2014, 50(14): 81-88. (in Chinese)

[13] R Ding, Z X Guo. Microstructural modelling of dynamic recrystallisation using an extended cellular automaton approach. Computational Materials Science, 2002, 23(1): 209-218.

[14] Chen, K Qi, Z Cui, et al. Modeling the dynamic recrystallization in austenitic stainless steel using cellular automaton method. Computational Materials Science, 2014, 83: 331-340.

[15] W Roberts, B Ahlblom. A nucleation criterion for dynamic recrystallization during hot working. Acta Metallurgica, 1978, 26(5): 801-813.

[16] Liu, B W Zhu, L X Li. Dynamic recrystallization of AZ31 magnesium alloysimulated by LaasraouiJonas dislocation equation coupled cellular automata method. The Chinese Journal of Nonferrous Metals, 2013, 23(4): 898-904

[17] F A Hua, Y S Yang, D Y Guo, et al. A grain growth cellular automata model based on the curvature-driven mechanism. Acta Metallurgica Sinica, 2004, 40(11): 1210-1214.

[18] X J Guan, XY Jiao, J J Zhou, et al. Cellular automata simulation of single grain growth. The Chinese Journal of Nonferrous Metals, 2007, 17(5): 699-703.

[19] Guo, Y Li, Z Guo, et al. Microstructural evolution of as-cast 42CrMo ring during hot rolling. Journal of Mechanical Engineering, 2014, 50(12): 30-35. (in Chinese)

[20] F Qin, Y Li, H Qi, et al. Deformation behavior and microstructure evolution of as-cast $42 \mathrm{CrMo}$ alloy in isothermal and non-isothermal compression. Journal of Materials Engineering and Performance, 2016, 25(11): 5040-5048.

[21] G Kugler, R Turk. Modeling the dynamic recrystallization under multistage hot deformation. Acta Materialia, 2004, 52(15): 4659-4668.

\section{Submit your manuscript to a SpringerOpen ${ }^{\circ}$ journal and benefit from:}

- Convenient online submission

- Rigorous peer review

- Open access: articles freely available online

- High visibility within the field

- Retaining the copyright to your article

Submit your next manuscript at springeropen.com 